\title{
SERUM ZINC LEVELS IN CHILDREN WITH FEBRILE SEIZURES
}

Srinivasa $\mathrm{S}^{1}$, Manjunath. M. N²

\section{HOW TO CITE THIS ARTICLE:}

Srinivasa S, Manjunath. M. N. "Serum Zinc Levels in Children with Febrile Seizures". Journal of Evolution of Medical and Dental Sciences 2014; Vol. 3, Issue 12, March 24; Page: 2983-2988,

DOI: $10.14260 /$ jemds/2014/2233

ABSTRACT: Febrile seizures are the most common cause of convulsions in children and a frequent cause of emergency hospital admissions. Indian studies suggested that up to $10 \%$ of children experience a febrile seizure. Febrile seizures are defined as an event in infancy or childhood usually occurring between 6 months to 6 years of age associated with fever but without evidence of intracranial infection or defined cause. OBJECTIVES: To determine the frequency of low serum zinc level in children presenting with febrile seizures at tertiary care hospital, Bangalore. METHOD: This is an observational cross sectional study conducted at the Department of Pediatric Medicine, tertiary care hospital, Bangalore, from January 2013 to January 2014. Children (6 months to 6 years of age) presenting with febrile seizures who satisfied inclusion and exclusion criteria were enrolled for the study. Cause of fever was determined after detailed history, physical examination and relevant investigations. Three milliliters centrifuged blood sample was preserved in acid washed test tube. Separated serum was used to measure serum zinc level by calorimetric method. RESULTS: Out of 100 children enrolled, male to female ratio was 1.4:1, 56\% of children were below the age of 2 years with mean age of the children was 24 months. Upper respiratory tract infection was the most frequent cause of fever apparent in 70 children (70\%), followed by dengue fever 11 children (11\%), acute gastroenteritis $6(6 \%)$, urinary tract infection and otitis media in 4 children each (8\%), Viral fever in 5 child (5\%). Frequency of low serum zinc level was $62 \%$ in children with febrile seizures. INTERPRETATION AND CONCLUSION: This study reveals that there is positive correlation between low serum zinc levels and febrile convulsions.

KEYWORDS: febrile convulsions, Epilepsy, Serum zinc, GABA, zinc supplementation.

INTRODUCTION: A Seizure is a paroxysmal time limited change in motor activity and or behavior that results from abnormal electrical activity in the brain. Less than one third of seizures in children are caused by epilepsy, a condition defined as two or more unprovoked seizures occurring at an interval greater than 24 hours apart. ${ }^{1}$ Epilepsy is one of the most common disorders of the brain. ${ }^{2}$

Worldwide, epilepsy affects 50 million people. According to a World Health Organization (WHO) survey, epilepsy accounts for $1 \%$ of the global burden of disease, a figure equivalent to breast cancer in women and lung cancer in men. ${ }^{3}$ Epilepsy is, of course, not a specific disease, or even a single syndrome, but rather a broad category of symptom complexes arising from any number of disordered brain functions that themselves may be secondary to a variety of pathologic processes. ${ }^{4}$

Febrile seizures are the most common type of seizures observed in pediatric age group. Febrile seizures is defined as an event in infancy or childhood usually occurring between six months to six years of age associated with fever but without evidence of intracranial infection or defined cause. ${ }^{5}$ Febrile seizures occurring before 6 months should raise the suspicion of serious infections like bacterial meningitis. Animal studies suggest a possible role of endogenous pyrogens such as interleukin $1 \beta$ that by increasing neuronal excitability, may link fever and seizures activity. ${ }^{6}$ 
Preliminary studies in children support the hypothesis that the cytokine network is activated and may have a role in pathogenesis of febrile seizures, but the precise clinical and pathological significance of these observations is not clear yet.7,8 Genetic influences may play a major role in febrile seizures. In a child with febrile seizures risk is $10 \%$ for sibling and increases to $50 \%$ for sibling if the parent has febrile seizures as well. ${ }^{9}$

TYPES OF FEBRILE SEIZURE: A simple febrile seizure is generalized, tonic clonic in nature, lasts for a few seconds and rarely up to $15 \mathrm{~min}$, is followed by a brief period of postictal drowsiness and occurs only once in 24 hrs. $^{1}$ A febrile seizure is described a complex or complicated when the duration is $>15 \mathrm{~min}$, when repeated convulsions occur within 24 hours, or when focal seizure activity or focal findings are present during the postictal period. ${ }^{1}$

About one third of children with a first febrile seizure experience recurrent seizures. ${ }^{10}$ Other risk factors are as follows ${ }^{11,12}$ younger age at time of febrile seizures, relatively low fever at time of first seizure, family history of febrile seizure in a first degree relative, brief duration between fever onset and initial seizure, multiple initial febrile seizures during the same episodes.

TRACE ELEMENTS IN FEBRILE SEIZURES: A number of trace elements are said to play a role in febrile convulsions by their co-enzyme activity or ability to influence ion channels and receptors.

Studies have shown that iron, zinc, selenium, copper and magnesium play a significant role in febrile convulsions. Zinc (Zn) acts as a co-factor of glutamic acid decarboxylase, an enzyme which maintains the production of GABA in central nervous system and decreased level of Zn in CSF has also been observed in febrile seizures.

Zinc increases the storage capacity of glutamate or slows the release rate of glutamate. Apart from this zinc also stimulates the activity of pyridoxal kinase, which is involved in the synthesis of pyridoxal phosphate from pyridoxal. Pyridoxal phosphate in turn stimulates the activity of glutamic acid decarboxylase which catalyzes GABA synthesis. Hence decrease in zinc concentration results in lowering of GABA level which can precipitate seizures. Recent evidences indicate that deficiency of zinc can play significant role in febrile seizures.

MATERIALS AND METHODS: This study was conducted during January 2013 to January 2014 in tertiary care hospital, Bangalore. Children between 6 months to 6 years of age presenting in Emergency department with fever $(\geq 38 \stackrel{\circ}{\circ}$ ) and history of seizures, having normal cerebrospinal fluid examination and normal; serum glucose, sodium, potassium, calcium and magnesium levels were included. The patients who had any evidence of central nervous system infection, epilepsy, metabolic seizures or those who are already on zinc therapy for any other ailment like malnutrition, diarrhea, pneumonia or acrodermatitis enteropathica were excluded.

Informed consent of parents of both the groups of children was obtained in a printed consent form. Any questions or doubts were cleared and signature of parent was obtained. The study protocol was approved by the ethics committee of our hospital.

Prior to inclusion of the children in the study, a detailed history of presenting complaints were recorded by our history included duration of fever, time of onset of seizures, type of seizures, duration of seizures, past and family history of seizures. In addition history suggestive of any triggering factors for febrile episode like cough, cold, nasal discharge, ear discharge, burning 
micturition or crying during micturition were also recorded. Vitals signs namely heart rate, respiratory rate, and blood pressure were measured and recorded. The axillary temperature was recorded in all children with mercury thermometer placed in axilla placed for three minutes.

Anthropometric measurements namely weight, height, mid-arm circumference and head circumference were recorded. This was followed by general examination and systemic examination in detail. Those children who showed features of any chronic congenital or acquired illness were excluded. Those who showed features of intracranial infection like altered sensorium, meningeal signs, bulging anterior fontanel etc. were also excluded.

Three milliliters of whole blood was collected by venepuncture under strict aseptic precautions and sent to biochemistry laboratory for assessment of serum zinc levels. Determination of serum zinc levels were done by calorimetric method. The principle being zinc in alkaline medium reacts with nitro PAPS to form purple coloured complex. Intensity of color formed is directly proportional to the amount of zinc present in the sample.

Though earlier studies have established a linear relationship between serum zinc and CSF zinc in children with febrile seizures, CSF zinc analysis was not done in our study and based on ethical grounds CSF analysis was not done in the children with fever alone.

RESULTS: Study design: A Cross sectional study.

Statistical software: The Statistical software SPSS 15.0 was used for the analysis of the data and Microsoft word and Excel have been used to generate graphs, tables etc.

Majority of children with febrile seizures (56\%) were below the age of two years. Mean age of children was 24 months. There were $60 \%$ of males with male to female ratio being 1.4:1. Frequency of low serum zinc levels were found in $62 \%$ of children (fig1).

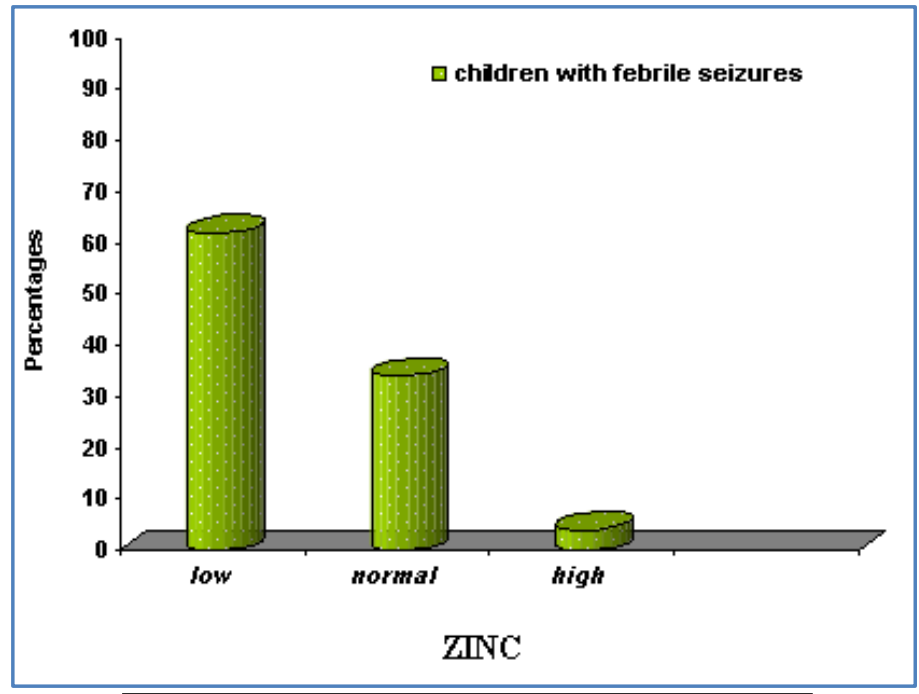

Fig 1: frequency of serum zinc levels

Fig 1: Serum zinc level-were low in $62 \%$ of cases of febrile seizures and normal in $34 \%$ of children. 
Frequency of low serum zinc levels with different age is shown in table1 and frequency of low serum zinc levels with reference to male to female is shown in table 2.

Febrile seizure children:

\begin{tabular}{|c|c|c|c|c|}
\hline Age & No. of cases & $\mathbf{\%}$ & Low zinc & $\mathbf{\%}$ \\
\hline$<2$ years & 56 & 56 & 38 & 67.8 \\
\hline 2-6 years & 44 & 44 & 24 & 54.5 \\
\hline Total & $\mathbf{1 0 0}$ & & $\mathbf{6 2}$ & \\
\hline
\end{tabular}

Table 1: Low Serum Zinc Level in Relation to Age

Febrile seizure children:

\begin{tabular}{|c|c|c|c|c|}
\hline Gender & No. of cases & \% & Low zinc & \% \\
\hline Male & 60 & 60 & 36 & 60 \\
\hline Female & 40 & 40 & 26 & 65 \\
\hline Total & $\mathbf{1 0 0}$ & & $\mathbf{6 2}$ & \\
\hline
\end{tabular}

Table 2: Low Serum Zinc Level in Relation to gender

Upper respiratory tract infection was most frequent cause of fever in $70 \%$ of cases followed by dengue fever (11\%), gastroenteritis in 6 children, otitis media and dengue fever in 4 children each and viral fever 5 children. Frequency of low serum zinc levels with reference to cause of fever is shown in table 3.

Febrile seizure children:

\begin{tabular}{|l|c|c|c|c|}
\hline Cause of fever & No. of cases & $\mathbf{\%}$ & Low zinc levels & \% \\
\hline Gastroenteritis & 6 & 6 & 2 & 33.3 \\
\hline Otitis media & 4 & 4 & 2 & 33.3 \\
\hline Dengue fever & 11 & 11 & 4 & 36.3 \\
\hline URTI & 70 & 70 & 52 & 74.2 \\
\hline UTI & 4 & 4 & 1 & 25 \\
\hline V. fever & 5 & 5 & 1 & 20 \\
\hline \multicolumn{2}{|c|}{ Total } & $\mathbf{1 0 0}$ & \multicolumn{4}{|c|}{$\mathbf{6 2}$} & \\
Table 3: Low Serum Zinc Level in Relation to cause of fever \\
\hline
\end{tabular}

DISCUSSION: Febrile seizure is a common neurologic problem occurring in children aged between 6 months to 6 years. The etiology of febrile seizure is unknown but genetic factors or electrolyte disturbances may have a role in its occurrence or recurrence. To date it is revealed that febrile seizures can be induced by several factors. It is generally believed that an FS is an age-dependent response of the immature brain to fever.

This postulation is supported by the fact that most (80-85\%) febrile seizures occur between 6 months and 3 years of age, with the peak incidence at 18 months. Although the mechanism of this 
increased susceptibility is unclear, animal models suggest that there is enhanced neuronal excitability during the normal brain maturation. Gamma amino butyric acid is an important inhibitory neurotransmitter.

Zinc has a regulatory effect on glutamic acid decarboxylase and the synthesis of GABA. Attempts have been made to identify predisposing risk factor like family history, metabolic disturbance (especially serum zinc, magnesium, glucose, calcium). This knowledge has a practical value and advising parents regarding recurrent convulsions.

This study was conducted at the tertiary care hospital, Bangalore. Age of presentation in majority of children (56\%) was 6 months to 2years. Frequency of low serum zinc level was found in $62 \%$ children with febrile seizures in this study, which is comparable to several other international studies done on febrile convulsions and zinc.

Mollah MA et al ${ }^{13}$ in 2008 published a study comparing serum and CSF Zinc levels of febrile seizure children to their matched non seizure febrile peers. Mean Zn concentration in both serum and CSF was less in febrile seizure children than in their matched non-seizure febrile peers $(p$ $<0.001$ ). AmiriM et al, ${ }^{14}$ Modarresi MR et al, ${ }^{15}$ Lee J and Kim JH, ${ }^{16}$ and Talebian A et al, ${ }^{17}$ also gave similar results which are comparable with our study.

The serum zinc levels did not show any significant correlation with age of onset, sex, axillary temperature or fever seizure interval in our study. All previous studies have shown similar findings in this aspect.

As serum zinc concentration in any population is influenced by factors such as dietary pattern, vitamin A, vitamin D deficiency, zinc levels in the soil and water, further studies are needed in this aspect to identify the probable cause for this finding.

CONCLUSION: This study shows that serum zinc levels are decreased in children with febrile convulsions, thus indicating that zinc deprivation plays significant role in the pathogenesis of febrile convulsions. The role of zinc in febrile convulsions should be investigated by further studies and if the results are reproducible, zinc supplementation can be given in febrile convulsions.

\section{REFERENCES:}

1. Nelson's Textbook of Pediatrics, P g 2013, nineteenth Edition.

2. World Health Organization. Atlas: Epilepsy Care in the World. Geneva: World Health Organization; 2005:91.

3. Johnston MV. Seizure in childhood: febrile seizure. 19th e d. In: Nelson's text book of pediatrics, Behrman RE, Kliegman RM, Stanton, eds. Pennsylvania: Saunders; 2004. pp. 2013-14.

4. Murray CJL, Lopez AD. Global Comparative Assessment in the Health Sector; Disease Burden, Expenditures, and Intervention Packages. Geneva: World Health Organization; 1994.

5. Consensus statement Febrile seizures: long term management of children with fever associated seizures, Pediatrics Dec 1980; (6):1009-12.

6. Matsuo $\mathrm{m}$ Sasaki $\mathrm{K}$ et al. Increased IL-IB production from leucocytes in febrile seizures. Pediatric Neurology Aug 2006; 35(2):102-6.

7. Gatti, Vezzani A. Mechanisms of fever and febrile seizures. Putative role of IL-1 system Febrile seizures San Diego, CA: academic press 2002:169-88 


\section{ORIGINAL ARTICLE}

8. Haspolat S, Mihci E et al. IL IB, TNF- alpha and nitrite levels in febrile seizures, J Child Neurolog Oct 2002:17(10):749-51

9. Frantzen, Lennox-Buchthal et al. A genetic study of febrile convulsions Neurology 1970, 20; 909-917.

10. Offringa M, Lubsen J, Nelson KB et al. Risk factors for seizure recurrence in children with febrile seizures: a pooled analysis from five studies. J Pediatric April 1994; 14(4):574-84.

11. Wriuru C, Appleton R. Febrile seizures and update. Arch Dis Child Aug 2004; 89(8):751-6.

12. Berg AT, Shinnar S, Shapiro. Predictors of recurrent febrile seizures A prospective cohort study, Arch Pediatr. Adolesc Med April 1997;151(4):371-8

13. Mollah Ranjan Dey, Tarafdar. Zinc in CSF of patients with febrile convulsions. Indian Journal of Paediatrics, Oct 2002, Vol 69, No 10, Pg 859-861.

14. Amiri M, Farzin L, Moassesi ME, Sajadi F. Serum trace element levels in febrile convulsion. Biol Trace Elem Res. 2010; 135:38-44.

15. Modarresi MR, Shahkarami SMA, Yaghini O, Shahbi J, Mosaiiebi D, Mahmoodian T. The relationship between zinc deficiency and febrile convulsion in Isfahan, Iran. Iranian J Child Neurol. 2011; 5:27-31.

16. Lee JH, Kim JH. Comparison of serum zinc levels measured by inductively coupled plasma mass spectrometry in preschool children with febrile and afebrile seizures. Ann Lab Med. 2012; 32:190-193.

17. Talebian A, Vakili Z, Talar SA, Kazemi SM, Mousavi GA. Assessment of the relation between serum zinc \& magnesium levels in children with febrile convulsion. Iranian J Pathol. 2009; 4:157-160.

\section{AUTHORS:}

1. Srinivasa S.

2. Manjunath. M. N.

\section{PARTICULARS OF CONTRIBUTORS:}

1. Professor, Department of Paediatrics, Kempegowda Institute of Medical Science and Research Centre, Bangalore.

2. Post Graduate, Department of Paediatrics, Kempegowda Institute of Medical Science and Research Centre, Bangalore.

\section{NAME ADDRESS EMAIL ID OF THE CORRESPONDING AUTHOR:}

Dr. Manjunath M. N,

Post Graduate, Department of Pediatrics, V. V. Puram, K. R. Road,

Bangalore - 560004, Karnataka.

E-mail: drmanju.drmanju@gmail.com

Date of Submission: 21/02/2014.

Date of Peer Review: 23/02/2014.

Date of Acceptance: 04/03/2014.

Date of Publishing: 18/03/2014. 